\title{
Target range of motion for rehabilitation after total knee arthroplasty
}

\author{
Hirotaka Mutsuzaki ${ }^{1}$, Ryoko Takeuchi ${ }^{1}$, Yuki Mataki ${ }^{1}$, and Yasuyoshi Wadano ${ }^{2}$ \\ ${ }^{I}$ Department of Orthopaedic Surgery, Ibaraki Prefectural University of Health Sciences, Japan \\ ${ }^{2}$ Department of Rehabilitation Medicine, Ibaraki Prefectural University of Health Sciences, Japan
}

\begin{abstract}
Objective: Postoperative knee range of motion (ROM) is among the most important factors influencing patient satisfaction after total knee arthroplasty (TKA). The purpose of this study was to clarify the time course of improvement in knee ROM up to 12 months after TKA, including intraoperative knee ROM after implantation, and to clarify a target ROM for rehabilitation after TKA.

Patients and Methods: In total, 39 knee joints in 26 patients with osteoarthritis who underwent TKA (retaining the posterior cruciate ligament) were evaluated. Goniometry was used to measure the knee range of extension and flexion preoperatively; intraoperatively; at 1 and 2 weeks after TKA; and then at 1, 3, 6, and 12 months after TKA.

Results: The postoperative extension range gradually improved up to a maximum at 6 months after TKA; there were no significant differences in the extension range between intraoperative and 6 months after TKA, intraoperative and 12 months after TKA, or 6 and 12 months after TKA. The postoperative flexion range gradually improved, with the maximum improvement observed at 3 months after TKA; there were no significant differences in the flexion range before TKA and 3, 6, and 12 months after TKA. There were no significant differences between flexion ROM measured at 3, 6, and 12 months after TKA.

Conclusions: The changes in the knee range of extension plateaued 6 months after TKA, and those in the knee range of flexion plateaued 3 months after TKA. The target range of extension for rehabilitation from 6 months to 12 months after TKA was the intraoperative range, and the target range of flexion for rehabilitation from 3 months to 12 months after TKA was the preoperative range.
\end{abstract}

Received: November 8, 2016

Accepted: January 10, 2017

Correspondence: Hirotaka Mutsuzaki, Department of Orthopaedic Surgery, Ibaraki Prefectural University of Health Sciences, 4669-2 Ami, Ami-machi, Inashiki-gun, Ibaraki 300-0394, Japan

E-mail: mutsuzaki@ipu.ac.jp

This is an open-access article distributed under the terms of the Creative Commons Attribution Non-Commercial No Derivatives (by-nc-nd) License $<$ https://creativecommons.org/licenses/by-nc-nd/4.0/>.
Key words: target range of motion, time course of improvement, total knee arthroplasty

(J Rural Med 2017; 12(1): 33-37)

\section{Introduction}

Total knee arthroplasty (TKA) has been widely implemented in recent years as a surgical treatment for osteoarthritis of the knee, and favorable long-term outcomes have been reported ${ }^{1-3)}$. Postoperative knee range of motion (ROM) is one of the most important factors influencing patient satisfaction after TKA. A limited flexion angle hinders activities of daily living, and patients experience low satisfaction levels when their activity is lower than the preoperative level or when they are unable to enjoy anticipated activities $^{4,5}$. It allows for better informed consent from patients during the preoperative explanation of TKA, and allows patients to have realistic expectations with regard to the outcome of the surgery. Moreover, an understanding of the time course of improvement in knee ROM after TKA will allow surgeons and rehabilitation therapists to monitor the progress of patients in a better manner, especially with respect to rehabilitation.

Few studies have reported the time course of improvement in knee ROM after TKA ${ }^{6,7)}$. The knee ROM reportedly reaches a plateau at 12 months after TKA ${ }^{6}$. However, the intraoperative knee ROM after implantation, knee ROM within 1 month after TKA, and the exact target ROM for rehabilitation after TKA remains unclear.

The purpose of this study was to clarify the time course of improvement in knee ROM for up to 12 months after TKA, including intraoperative knee ROM after implantation, and to clarify a target ROM for rehabilitation after TKA. The hypothesis was that there is a time point at which a target ROM for rehabilitation is reached after TKA. 


\section{Patients and Methods}

The ethics committee of Ibaraki Prefectural University of Health Sciences reviewed and approved the study (No. 664). Informed consent was obtained from each patient.

From July 2009 to August 2015, unilateral and bilateral primary TKA (retaining the posterior cruciate ligament) was performed on 40 joints in 27 patients by a senior surgeon (H.M.). Those bilateral TKAs were not simultaneous operations. One patient was dropped from the study because of transfer to a different hospital; ultimately, a total of 39 joints in 26 patients were evaluated in this study. All patients had medial osteoarthritis (OA) of the knee. The preoperative characteristics, including age, sex, height, weight, body mass index, preoperative femorotibial angle, operative time, implant installation angle ${ }^{8}$, and interval between surgery and hospital discharge are summarized in Table 1.

All patients were administered general anesthesia. We made a midline skin incision, and used a medial parapatellar approach. We used an intramedullary alignment rod for femoral cutting, and an extramedullary guide system for tibial cutting using an independent cut technique. We routinely grafted a bone plug in the femoral canal for intramedullary guidance. We did not replace the patella, and retained the posterior cruciate ligament. We fixed components without cement, except when we used metal block implants, for which cement fixation is recommended, and when the bone was osteoporotic. We used the following implants: the Scorpio NRG CR HA (Stryker Howmedica Osteonics, Allendale, NJ, USA) in 6 knees, the LFA CR (JMM, Osaka, Japan) in 2 knees, and the NexGen CR (Zimmer, Warsaw, IN, USA) in 31 knees. With the NexGen CR type, the femoral component was the HA-TCP CR Femoral or CR-flex Porus Femoral. The tibial component was the NexGen CR HA-TCP Stem Tibia or NexGen Trabecular Metal Monoblock Tibia. After fixing the components, we confirmed the stability of the ligament balance.

To reduce blood loss, intra-articular tranexamic acid (10\% Transamin, 10 ml, 1,000 mg; Daiichi-Sankyo, Tokyo, Japan) was injected via the drain in a retrograde manner after the wound was closed, and the drain was then clamped for 1 hour ${ }^{9}$. We used an intra-articular drain connected to a vacuum bag.

Postoperatively, all patients received intravenous prophylactic antibiotic therapy consisting of $1 \mathrm{~g}$ of cefazolin every 12 hours for 3 days. Standard thromboprophylaxis was also prescribed for all patients postoperatively. We used a foot pump (Novamedix A-V Impulse System; Kobayashi Medical, Osaka, Japan) and antiembolic stockings (Ansilk®; ALCARE, Tokyo, Japan) for thromboembolic prophylaxis $^{10,11)}$.
Table 1 Patient characteristics

\begin{tabular}{|c|c|c|}
\hline \multicolumn{3}{|l|}{ Characteristic } \\
\hline \multicolumn{2}{|l|}{ Patient (n) } & 26 \\
\hline \multicolumn{2}{|l|}{ Age (year) } & $76.7 \pm 6.2$ \\
\hline \multicolumn{2}{|l|}{ Sex (male/female) } & $8 / 18$ \\
\hline \multicolumn{2}{|l|}{ Disease } & $\begin{array}{c}\text { Osteoarthri- } \\
\text { tis }\end{array}$ \\
\hline \multicolumn{2}{|l|}{ Height $(\mathrm{cm})$} & $152.4 \pm 8.5$ \\
\hline \multicolumn{2}{|l|}{ Body weight (kg) } & $60.9 \pm 11.1$ \\
\hline \multicolumn{2}{|l|}{ BMI $\left(\mathrm{kg} / \mathrm{m}^{2}\right)$} & $26.1 \pm 3.8$ \\
\hline \multicolumn{2}{|l|}{ Knee (n) } & 39 \\
\hline \multicolumn{2}{|l|}{ Preoperative FTA $\left(^{\circ}\right)$} & $185.6 \pm 4.3$ \\
\hline \multicolumn{2}{|l|}{ Operative time } & $90.4 \pm 18.0$ \\
\hline \multicolumn{3}{|c|}{ Implant installation angle } \\
\hline & $\alpha\left(^{\circ}\right)$ & $83.7 \pm 1.4$ \\
\hline & $\beta\left(^{\circ}\right)$ & $90.2 \pm 1.3$ \\
\hline & $\gamma\left({ }^{\circ}\right)$ & $3.7 \pm 2.5$ \\
\hline & $\delta\left({ }^{\circ}\right)$ & $84.2 \pm 2.3$ \\
\hline Time from the operat & e (day) & $83.8 \pm 47.3$ \\
\hline
\end{tabular}

Results are represented as the mean \pm SD. BMI: body mass index, FTA: femorotibial angle.

We removed sutures of all the patients 2 weeks postoperatively. A continuous passive movement machine, and active and passive ROM therapy by a physical therapist commenced on postoperative Day 2. One week after TKA, patients were allowed to stand, and walk with full weight bearing using crutches, a walker, or a cane, as required. Hospitalized rehabilitation comprised 40 to 60 minutes of physical therapy and 40 to 60 minutes of occupational therapy. Patients were rehabilitated once a week for 3 months after discharge from the hospital.

We measured the knee range of extension and flexion before TKA; intraoperatively; at 1 and 2 weeks after TKA; and at $1,3,6$, and 12 months after TKA. Knee ROM was measured using goniometry, accurate to $1^{\circ}$. The landmarks used in the measurements were the greater trochanter of the femur, the proximal head of the fibula, and the lateral malleolus. In addition, the Japanese Orthopaedic Association OA knee rating score (JOA score) ${ }^{12)}$ was recorded before and 12 months after TKA. The JOA score rates pain on walking (0-30 points), pain on ascending and descending the stairs ( $0-25$ points), ROM ( $0-35$ points), and joint swelling $(0-30$ points) for patients with OA, with a maximum total score of 100 points. Strong correlations were observed between JOA score and Knee Society function score ${ }^{13,14)}$. The JOA score at 12 months was significantly greater than the preoperative JOA score (79.1 \pm 9.7 vs. $52.6 \pm 7.8, p<0.001$, paired t-test).

ROM data were analyzed using a one-way repeated measure analysis of variance. As a post-hoc analysis, the Bonferroni comparisons test was performed. A $p$ value of $<$ 


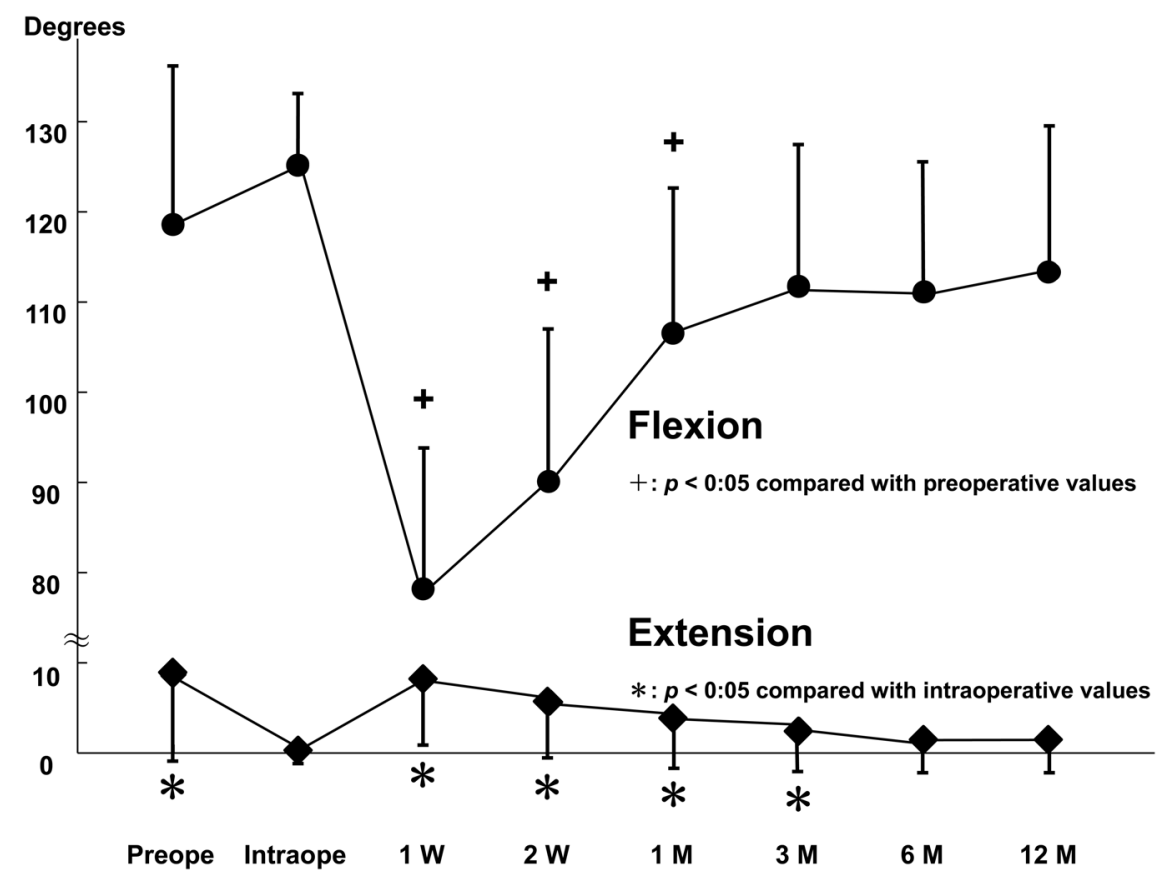

Figure 1 Graph showing the range of knee flexion and extension over time. Upper graph shows the range of flexion. Lower graph shows the range of extension. Extension angle is shown as the flexion angle.

0.05 was considered statistically significant. Analyses were performed using IBM SPSS Statistics (Version 22; SPSS, Chicago, IL, USA).

\section{Results}

The mean values of range of extension and flexion at the various time points are summarized in Table 2. The values of range of extension and flexion angle are described and plotted in Table 2 and Figure 1.

There were no significant differences in the range of extension between intraoperative and 6 months after TKA ( $p=$ $0.293)$, intraoperative and 12 months after TKA $(p=0.652)$, and between 6 and 12 months after TKA $(p=1.000)$. There were no significant differences in the range of extension between 3 and 6 months after TKA $(p=1.000)$, and between 3 and 12 months after TKA $(p=1.000)$; however, the range of extension at 3 months after TKA was significantly greater than the intraoperative range of extension $(p=0.040)$. Compared with the preoperative range of extension, there were significant differences between that measured intraoperatively $(p<0.001)$, and that measured $3(p=0.029), 6(p<$ $0.001)$, and 12 months after TKA $(p<0.001)$. The postoperative range of extension gradually improved in comparison with the preoperative extension range, with the maximum improvement observed at 6 months after TKA (Figure 1).
Table 2 Range of motion (ROM)

\begin{tabular}{llcc}
\hline & Time point & ROM $\left(^{\circ}\right)$ & $p$ value \\
\hline Extension & preoperative & $8.7 \pm 9.6$ & $p<0.001$ \\
& intraoperative & $0.3 \pm 1.1$ & NA \\
& 1 week & $8.3 \pm 7.3$ & $p<0.001$ \\
& 2 weeks & $5.8 \pm 6.0$ & $p<0.001$ \\
& 1 month & $3.9 \pm 5.4$ & $p=0.001$ \\
& 3 months & $2.7 \pm 4.7$ & $p=0.029$ \\
& 6 months & $1.5 \pm 3.5$ & NS \\
& 12 months & $1.5 \pm 3.8$ & NS \\
& preoperative & $118.5 \pm 18.3$ & NA \\
& intraoperative & $125.0 \pm 7.2$ & $p=0.413$ \\
& 1 week & $78.2 \pm 15.6$ & $p<0.001$ \\
& 2 weeks & $90.6 \pm 15.3$ & $p<0.001$ \\
& 1 month & $106.8 \pm 15.8$ & $p=0.015$ \\
& 3 months & $112.1 \pm 14.8$ & NS \\
& 6 months & $111.5 \pm 13.7$ & NS \\
& 12 months & $113.6 \pm 15.3$ & NS
\end{tabular}

Results are represented as the mean $\pm \mathrm{SD}$. $p$ values for extension range compared with intraoperative values. $p$ values for flexion range compared with preoperative values. NS: not significant, NA: not applicable. Extension angle is shown as the flexion angle.

There were no significant differences between the range of flexion before TKA and 3 months after TKA $(p=1.000)$, before TKA and 6 months after TKA $(p=0.728)$, before 
TKA and 12 months after TKA ( $p=1.000)$, between 3 and 6 months after TKA $(p=1.000)$, between 3 months and 12 months after TKA ( $p=1.000)$, and between 6 and 12 months after TKA $(p=1.000)$. The intraoperative range of flexion was significantly greater than that at 1 week $(p<0.001)$, 2 weeks $(p<0.001), 3$ months $(p<0.001), 6$ months $(p<$ $0.001)$, and 12 months after TKA ( $p=0.001)$; however, there were no significant differences between the intraoperative range of flexion and preoperative flexion range $(p=0.413)$. The postoperative range of flexion gradually improved, with the maximum improvement observed at 3 months after TKA (Figure 1).

\section{Discussion}

The main findings of the current study were that changes in the knee range of extension plateaued at 6 months after TKA, and those in the knee range of flexion plateaued at 3 months after TKA. The target range of knee extension for rehabilitation from 6 months to 12 months after TKA was the intraoperative extension range, and the target range of flexion for rehabilitation from 3 months to 12 months after TKA was the preoperative flexion range. Although the postoperative range of extension gradually improved in comparison with the preoperative range, with the maximum improvement observed at 6 months after TKA, the intraoperative range of extension was significantly lesser than that at 3 months after TKA; there were no significant differences between the extension range measured intraoperatively and 6 months after TKA, intraoperatively and 12 months after TKA, and between 6 and 12 months after TKA. The postoperative range of flexion gradually improved, with the maximum improvement observed at 3 months after TKA; there were no significant differences between the range of flexion before TKA and 3, 6, and 12 months after TKA, between 3 and 6 months after TKA, between 3 and 12 months after TKA, and between 6 and 12 months after TKA.

The postoperative flexion angle is reportedly positively correlated with the preoperative flexion angle ${ }^{15,16)}$. Preoperative soft tissue condition can influence the postoperative flexion angle. The current study found similar pre- and postoperative flexion angles, which is similar to the results of previous reports ${ }^{15,16}$. Therefore, the target range of flexion for rehabilitation after TKA can be set as the preoperative flexion angle. In contrast, the postoperative knee extension angle is reportedly significantly greater than the preoperative levels ${ }^{6}$. We clarified the target extension angle for rehabilitation after TKA for the first time. Although the extension angle is corrected by bone cutting and intraoperative soft tissue release, the ROM is reportedly insufficient for 1 month after TKA owing to decreased quadriceps strength and postoperative pain ${ }^{17)}$. Zhou et al. reported that the knee ROM reached a plateau at 12 months after $\mathrm{TKA}^{6}$. In the current study, earlier recovery of the flexion (3 months) and extension ranges (6 months) were observed compared to findings reported in a previous study ${ }^{6}$. This may be because of possible mild swelling after TKA in the current study due to tranexamic acid being used to minimize bleeding. In addition, our patients underwent several rehabilitation sessions owing to the long duration of hospitalization. Prospective randomized studies are necessary to clarify the effect of tranexamic acid use and long duration of hospitalization.

The difference in ROM between posterior-stabilized implants and cruciate-retaining implants has been studied well. Several studies have reported that posterior-stabilized implants achieved a slightly higher degree of flexion than that achieved by cruciate-retaining implants ${ }^{18-20)}$, while at least 1 study has found no significant difference in the ROM between posterior-stabilized implants and cruciate-retaining implants ${ }^{21}$. In the current study, we used only cruciateretaining implants. In the future, it may be necessary to compare the change in ROM at several time points between patients with cruciate-retaining implants versus those with posterior-stabilized implants.

Patients reportedly have low satisfaction with a limited postoperative flexion range when their activity is even lower than it was before TKA, or when they are unable to enjoy anticipated activities ${ }^{4,5,22)}$. Predicting postoperative ROM is complicated because the results can be affected by multiple factors such as the age and sex of the patient, diagnosis, preoperative ROM, the skill of the operating surgeon, design of the prosthesis, and postoperative rehabilitation ${ }^{15,23,24)}$. Clinically, accurate prediction of postoperative ROM allows for better informed patient consent during preoperative discussions. Knowledge of the time course of improvement in knee ROM after TKA will allow patients to have realistic expectations of the surgical outcome. Moreover, an understanding of the time course of improvement in knee ROM after TKA will enable surgeons and rehabilitation therapists to monitor the progress of the patients in a better manner.

The current study has potential limitations. First, unlike this study, a prospective randomized study designs achieve higher degrees of evidence. Second, the number of patients was small, and the results might be different from those of studies with larger sample sizes. Third, the follow-up period was only 12 months. Moreover, no data were collected between 6 and 12 months after TKA; however, the knee ROM did not change significantly with regard to the flexion or extension range during this period after TKA. A long-term follow-up study with short interval investigation time points is warranted. Finally, the implant types were not unified; unification of the implant type may be necessary. 


\section{Conclusions}

The changes in the range of extension of the knee plateaued at 6 months after TKA, and those in the knee range of flexion of the knee plateaued at 3 months after TKA. The target range of extension for rehabilitation from 6 months to 12 months after TKA was the intraoperative extension range, and the target range of flexion for rehabilitation from 3 months to 12 months after TKA was the preoperative flexion range.

Conflicts of Interest: The authors declare no conflict of interest.

\section{References}

1. Martin A, Quah C, Syme G, et al. Long term survivorship following Scorpio Total Knee Replacement. Knee 2015; 22: 192-196. [Medline] [CrossRef]

2. Hack J, Mai S, Siebert W. 10-year follow-up of the NexGen CR total knee prosthesis. Z Orthop Unfall 2015; 153: 508515 (in German). [Medline]

3. Mont MA, Pivec R, Issa K, et al. Long-term implant survivorship of cementless total knee arthroplasty: a systematic review of the literature and meta-analysis. J Knee Surg 2014; 27: 369-376. [Medline]

4. Matsuda S, Kawahara S, Okazaki K, et al. Postoperative alignment and ROM affect patient satisfaction after TKA. Clin Orthop Relat Res 2013; 471: 127-133. [Medline] [CrossRef]

5. Noble PC, Conditt MA, Cook KF, et al. The John Insall Award: Patient expectations affect satisfaction with total knee arthroplasty. Clin Orthop Relat Res 2006; 452: 35-43. [Medline] [CrossRef]

6. Zhou Z, Yew KS, Arul E, et al. Recovery in knee range of motion reaches a plateau by 12 months after total knee arthroplasty. Knee Surg Sports Traumatol Arthrosc 2015; 23: 1729-1733. [Medline] [CrossRef]

7. Springorum HR, Maderbacher G, Craiovan B, et al. No difference between standard and high flexion cruciate retaining total knee arthroplasty: a prospective randomised controlled study. Knee Surg Sports Traumatol Arthrosc 2015; 23: 15911597. [Medline] [CrossRef]

8. Ewald FC. The Knee Society total knee arthroplasty roentgenographic evaluation and scoring system. Clin Orthop Relat Res 1989; 9-12. [Medline]

9. Mutsuzaki H, Ikeda K. Intra-articular injection of tranexamic acid via a drain plus drain-clamping to reduce blood loss in cementless total knee arthroplasty. J Orthop Surg 2012; 7: 32. [Medline] [CrossRef]

10. Morris RJ, Woodcock JP. Evidence-based compression: prevention of stasis and deep vein thrombosis. Ann Surg 2004; 239: 162-171. [Medline] [CrossRef]

11. Stannard JP, Harris RM, Bucknell AL, et al. Prophylaxis of deep venous thrombosis after total hip arthroplasty by using intermittent compression of the plantar venous plexus. Am J Orthop 1996; 25: 127-134 (Belle Mead NJ). [Medline]

12. The Japanese Orthopaedic Association Japanese Knee Society. Assessment Criteria for Knee Diseases and Treatments. Tokyo: Kanehara 1994.

13. Insall JN, Dorr LD, Scott RD, et al. Rationale of the Knee Society clinical rating system. Clin Orthop Relat Res 1989; 13-14. [Medline]

14. Sasaki E, Tsuda E, Yamamoto Y, et al. Relationship between patient-based outcome score and conventional objective outcome scales in post-operative total knee arthroplasty patients. Int Orthop 2014; 38: 373-378. [Medline] [CrossRef]

15. Gatha NM, Clarke HD, Fuchs R, et al. Factors affecting postoperative range of motion after total knee arthroplasty. J Knee Surg 2004; 17: 196-202. [Medline]

16. Ritter MA, Harty LD, Davis KE, et al. Predicting range of motion after total knee arthroplasty. Clustering, log-linear regression, and regression tree analysis. J Bone Joint Surg Am 2003; 85-A: 1278-1285. [Medline] [CrossRef]

17. Mizner RL, Petterson SC, Snyder-Mackler L. Quadriceps strength and the time course of functional recovery after total knee arthroplasty. J Orthop Sports Phys Ther 2005; 35: 424-436. [Medline] [CrossRef]

18. Harato K, Bourne RB, Victor J, et al. Midterm comparison of posterior cruciate-retaining versus -substituting total knee arthroplasty using the Genesis II prosthesis. A multicenter prospective randomized clinical trial. Knee 2008; 15: 217-221. [Medline] [CrossRef]

19. Jacobs WC, Clement DJ, Wymenga AB. Retention versus removal of the posterior cruciate ligament in total knee replacement: a systematic literature review within the Cochrane framework. Acta Orthop 2005; 76: 757-768. [Medline] [CrossRef]

20. Maruyama S, Yoshiya S, Matsui N, et al. Functional comparison of posterior cruciate-retaining versus posterior stabilized total knee arthroplasty. J Arthroplasty 2004; 19: 349-353. [Medline] [CrossRef]

21. Ishii Y, Noguchi H, Takeda M, et al. Prediction of range of motion 2 years after mobile-bearing total knee arthroplasty: PCL-retaining versus PCL-sacrificing. Knee Surg Sports Traumatol Arthrosc 2011; 19: 2002-2008. [Medline] [CrossRef]

22. Choi HR, Siliski J, Malchau H, et al. How often is functional range of motion obtained by manipulation for stiff total knee arthroplasty? Int Orthop 2014; 38: 1641-1645. [Medline] [CrossRef]

23. Harvey IA, Barry K, Kirby SP, et al. Factors affecting the range of movement of total knee arthroplasty. J Bone Joint Surg Br 1993; 75: 950-955. [Medline]

24. Schurman DJ, Matityahu A, Goodman SB, et al. Prediction of postoperative knee flexion in Insall-Burstein II total knee arthroplasty. Clin Orthop Relat Res 1998; 175-184. [Medline] [CrossRef] 